\title{
BUYING AND SELLING RULES FOR A SIMPLE TRANSACTION OF A MEAN-REVERTING ASSET
}

\author{
DONG-HOON SHIN
}

\begin{abstract}
We consider an optimal trading rule in this paper. We assume that the underlying asset follows a mean-reverting process and the transaction consists of one buying and one selling. To maximize the profit, we find price levels to buy low and to sell high. Associated HJB equations are used to formulate the value function. A verification theorem is provided for sufficient conditions. We conclude the paper with a numerical example.
\end{abstract}

\section{INTRODUCTION}

A simplest trading strategy in financial market is to buy law and to sell high a share. However, it is controvertial to decide appropriate price levels of the share to buy or sell in practical market. This paper contributes to find the price levels of the share when it is governed by a mean-reverting model. A mean reversion process is one of the often and widely used models in financial markets to formulate the movement of an asset price that tends to converge an equibrilium of the prices. Poterba and Summers [9] applied variance ratio test to detect mean reversion in stock prices and market returns for US and seventeen countries. Frankel and Rose [4] examined derivations from purchasing power parity to show strong evidence of meanreversion in real exchange rates of various countries. Fama and French [3] tested a standard economic argument that profitability is mean reverting. A mean reversion process, or Ornstein-Uhlenbeck process (named after Ornstein and Uhlenbeck [8]), has a drift term that brings the variable being modeled back to an equilibrium level. The variable tends to oscillate around this equilibrium.

Consider the price of stock $S(t)=e^{X(t)}$ where $X(t)$ is the mean-reversion process which satisfies the following:

Received by the editors December 24, 2010. Revised April 4, 2011. Accepted April 19, 2011. 2000 Mathematics Subject Classification. 91G80.

Key words and phrases. mean-reversion, regime switching, optimal stopping. 


$$
d X(t)=a(b-X(t)) d t+\sigma d w(t),
$$

where $a$ is the rate of reversion, $b$ is the equilibrium level, $\sigma$ is the volatility, and $w(t)$ is a standard Brownian motion. We consider a trading rule consisting of buying and selling a share.

The problem is to find good price levels to buy and sell the share. We aim at maximizing our profit through transactions. A basic strategy is to buy low and sell high. However, picking an appropriate buying and selling price levels is difficult in practice.

Prior to this study, Guo and Zhang [2] used a smooth-fit technicque to characterize the optimal selling rule under a model with hybrid GMB. Zhang and Zhang [10] studied the problem finding the buying and selling levels assuming infinitively buying and selling. Song, Yin and Zhang [7] calculated the level pairs with a stochastic approximation methods.

In this article, we consider the trading rule that allows a round trip transactions, i.e., only buy once and sell once. Let the thresholds pair $\left(x_{1}, x_{2}\right)$ be the pair of buying price level and selling price level. We will determine the value of $\left(x_{1}, x_{2}\right)$ based on the parameters, $a, b, \sigma$, a discount factor $\rho$, and transaction discount factor $K$.

The rest of this paper is arranged as follows. Section 2 begins with the mean reversion model and the setup of the problem. Section 3 presents some properties of the value functions. Section 4 introduces a verification theorem which demonstrates the optimality of the trading rule. Section 5 includes numerical examples to show the dependency of $\left(x_{1}, x_{2}\right)$ and values of value functions on various parameters. In the last section, we compare the result of [10] with our results.

\section{Problem Formulation}

Let $X(t) \in \mathbb{R}$ denote a mean-reverting process governed by

$$
d X(t)=a(b-X(t)) d t+\sigma d w(t), \quad X(0)=x
$$

where $a>0$ is the rate of reversion, $b$ is the equilibrium level, $\sigma>0$ is the volatility, and $w(t)$ is a standard Brownian motion. Let

$$
0 \leq \tau \leq \delta
$$

denote stopping times of a buying decision $\tau$ and a selling decision $\delta$. The net position is flat(no stock holding) at $t \in[0, \tau) \cup[\delta, \infty)$ and long(one share of stock 
holding) at $t \in[\tau, \delta)$.

The asset price is given by $S(t)=\exp X(t)$. Suppose that $0<K<1$ is the percentage of slippage or commission per transaction and the discount factor $\rho>0$. We aim to maximize the reward functions

$$
\begin{aligned}
& J_{1}\left(x, \Lambda_{1}\right)=E\left[e^{-\rho \delta} S(\delta)(1-K)-e^{-\rho \tau} S(\tau)(1+K)\right], \\
& J_{2}\left(x, \Lambda_{2}\right)=E\left[e^{-\rho \delta} S(\delta)(1-K)\right]
\end{aligned}
$$

where $\Lambda_{1}=\{\tau, \delta\}$ and $\Lambda_{2}=\{\delta\}$. For $i=1,2$, let $V_{i}(x)$ be the value functions with $X(0)=x$ and initial net position $i$. Therefore, we have

$$
V_{i}(x)=\sup _{\Lambda_{i}} J_{i}\left(x, \Lambda_{i}\right)
$$

\section{Properties of the Value Functions}

In this section, we discuss various properties of the value functions and solve the stopping problem with a smooth fit principle. Note that

$$
V_{1}(x) \geq J_{1}\left(x, \Lambda_{1}\right)=J_{2}\left(x, \Lambda_{2}\right)-E e^{-\rho \tau} S(\tau)(1+K)
$$

In particular, setting $\tau=0$, we have the inequality

$$
V_{1}(x) \geq V_{2}(x)-e^{x}(1+K) .
$$

Otherwise, we similarly obtain

$$
V_{2}(x) \geq e^{x}(1-K) .
$$

The next lemma guarantees the bounds of $V_{i}(x)$.

Lemma 3.1. There exist constants $K_{1}, K_{2}$ and $K_{3}$ such that

$$
\begin{aligned}
& 0 \leq V_{1}(x) \leq K_{1} \text { and } \\
& 0 \leq V_{2}(x) \leq K_{2} e^{x}+K_{3} .
\end{aligned}
$$

Proof. It is clear that $V_{i}(x)$ are nonnegative by definition. We first find the upper bounds. From Dynkin's formula, we have

$$
E e^{-\rho \delta} S(\delta)-E e^{-\rho \tau} S(\tau)=E \int_{\tau}^{\delta} e^{-\rho t} e^{X(t)}(A-a X(t)) d t
$$

where $A=\sigma^{2} / 2+a b-\rho$. Note that $e^{x}(A-a x)$ is bounded above on $\mathbb{R}$. Let $C$ be an upper bound. It follows that

$$
E e^{-\rho \delta} S(\delta)-E e^{-\rho \tau} S(\tau) \leq C E \int_{\tau}^{\delta} e^{-\rho t} d t
$$




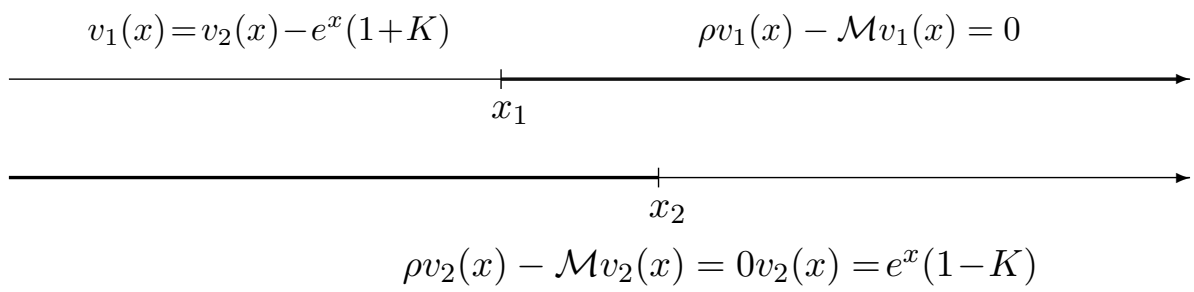

Figure 1. Thicken lines are Continuation Regions

Using the definition of $J_{1}\left(x, \Lambda_{1}\right)$, we have

$$
\begin{aligned}
J_{1}\left(x, \Lambda_{1}\right) & \leq E e^{-\rho \delta} S(\delta)-E e^{-\rho \tau} S(\tau) \\
& \leq C E \int_{\tau}^{\delta} e^{-\rho t} d t \\
& \leq C \int_{0}^{\infty} e^{-\rho t} d t=\frac{C}{\rho}:=K_{1}
\end{aligned}
$$

This implies that $0 \leq V_{1}(x) \leq K_{1}$.

Similarly, we have the inequality

$$
J_{2}\left(x, \Lambda_{2}\right) \leq E e^{-\rho \delta} S(\delta)(1-K) .
$$

As in (4), we can take $\tau=0$ and show that

$$
E e^{-\rho \delta} e^{X(\delta)}-e^{x} \leq \frac{C}{\rho} .
$$

It implies that

$$
V_{2}(x) \leq(1-K)\left(e^{x}+\frac{C}{\rho}\right):=K_{2} e^{x}+K_{3} .
$$

This completes the proof.

Let $\mathcal{M}$ denote the generator of $X(t)$. That is,

$$
\mathcal{M}=a(b-x) \frac{\partial}{\partial x}+\frac{\sigma^{2}}{2} \frac{\partial^{2}}{\partial x^{2}} .
$$

The associated HJB equations have the form:

$$
\left\{\begin{array}{l}
\min \left\{\rho v_{1}(x)-\mathcal{M} v_{1}(x), v_{1}(x)-v_{2}(x)+e^{x}(1+K)\right\}=0 \\
\min \left\{\rho v_{2}(x)-\mathcal{M} v_{2}(x), v_{2}(x)-e^{x}(1-K)\right\}=0
\end{array}\right.
$$

Recall that we decide to buy when the price is low and sell when the price is high. We say the low price as $S_{1}$ and the high price as $S_{2}$. Let $x_{j}=\log \left(S_{j}\right), j=1,2$. Then the continuation region for $i=1$ should be $\left(x_{1}, \infty\right)$ on which $\rho v_{1}(x)-\mathcal{M} v_{1}(x)=0$. If $x<x_{1}, v_{1}(x)=v_{2}(x)-e^{x}(1+K)$. Similarly, the continuation region for $i=2$ should be $\left(-\infty, x_{2}\right)$ on which $\rho v_{2}(x)-\mathcal{M} v_{2}(x)=0$. If $x>x_{2}, v_{2}(x)=e^{x}(1-K)$. 
Note that we need two restrictions for the values of $x_{1}$ and $x_{2}$ from the HJB equation. The stock trade, buying at $S_{1}=e^{x_{1}}$ and selling at $S_{2}=e^{x_{2}}$, is expected to be positive with the slippage cost. Therefore, it implies $e^{x_{2}}(1-K)>e^{x_{1}}(1+K)$, i.e.,

$$
x_{2}-x_{1}>\log \frac{1+K}{1-K} .
$$

In addition, $(\rho-\mathcal{M}) v_{2}(x)=0$ on $\left(-\infty, x_{1}\right)$. Since $v_{1}(x)=v_{2}(x)-e^{x}(1+K)$, and $(\rho-\mathcal{M}) v_{1}(x) \geq 0$ on $\left(-\infty, x_{1}\right)$, we have $(\rho-\mathcal{M}) e^{x} \leq 0$ on $\left(-\infty, x_{1}\right)$. Similarly, since $v_{2}(x)=e^{x}(1-K)$, and $(\rho-\mathcal{M}) v_{2}(x) \geq 0$ on $\left(x_{2}, \infty\right)$, we have $(\rho-\mathcal{M}) e^{x} \geq 0$ on $\left(x_{2}, \infty\right)$. These implies

$$
x_{1} \leq \frac{1}{a}\left(\frac{\sigma^{2}}{2}+a b-\rho\right) \leq x_{2} .
$$

For the next step, we solve the solution $\rho v_{i}(x)-\mathcal{M} v_{i}(x)=0$ with $i=1,2$. Let $\beta=\sqrt{2 a} / \sigma$ and $\eta(t)=t^{\rho / a-1} \exp \left(-t^{2} / 2\right)$. Then the general solution is given by

$$
C_{1} \int_{0}^{\infty} \eta(t) e^{-\beta(b-x) t} d t+C_{2} \int_{0}^{\infty} \eta(t) e^{\beta(b-x) t} d t
$$

for some constants $C_{1}, C_{2}$. (See [1] for the detail.) In view of the above lemma, $v_{2}(-\infty)$ should be bounded which implies $C_{2}=0$. Similarly, the bound of $v_{1}(\infty)$ implies $C_{1}=0$. Therefore, we have

$$
\begin{aligned}
& v_{1}(x)=C_{2} \int_{0}^{\infty} \eta(t) e^{\beta(b-x) t} d t \\
& v_{2}(x)=C_{1} \int_{0}^{\infty} \eta(t) e^{-\beta(b-x) t} d t .
\end{aligned}
$$

Note that both $v_{1}$ and $v_{2}$ are $C^{2}$ functions on $\left(x_{1}, \infty\right)$ and $\left(-\infty, x_{2}\right)$, respectively. Therefore, we apply the smooth fit principle at $x_{1}$ and $x_{2}$ which requires

$$
\left\{\begin{array}{l}
v_{1}\left(x_{1}\right)=v_{2}\left(x_{1}\right)-e^{x_{1}}(1+K), \\
v_{1}^{\prime}\left(x_{1}\right)=v_{2}^{\prime}\left(x_{1}\right)-e^{x_{1}}(1+K), \\
v_{2}\left(x_{2}\right)=e^{x_{2}}(1-K), \\
v_{2}^{\prime}\left(x_{2}\right)=e^{x_{2}}(1-K) .
\end{array}\right.
$$

From the $v_{2}(x)$ of $(7)$, we have $v_{2}\left(x_{2}\right)=v_{2}^{\prime}\left(x_{2}\right)$. Combining $v_{2}(x)$ in $(6)$, we get an equation for $x_{2}$ such that

$$
\int_{0}^{\infty} \eta(t) e^{-\beta\left(b-x_{2}\right) t} d t=\int_{0}^{\infty} \eta(t) \beta t e^{-\beta\left(b-x_{2}\right) t} d t .
$$

Moreover, it is easy to find $C_{1}$ by plugging $x_{2}$ into the $v_{2}(x)$ equation on $(7)$ and finally we get $v_{2}(x)$ on $\left(-\infty, x_{2}\right)$. 
After the $v_{2}(x)$ is determined from an explicit $x_{2}$ and $C_{1}$, we use it to solve $v_{1}(x)$. In particular, the combination of (6) and (7) gives the following:

$$
\left\{\begin{array}{l}
C_{2} \int_{0}^{\infty} \eta(t) e^{\beta\left(b-x_{1}\right) t} d t=C_{1} \int_{0}^{\infty} \eta(t) e^{-\beta\left(b-x_{1}\right) t} d t-e^{x_{1}}(1+K), \\
C_{2} \int_{0}^{\infty}-\beta t \eta(t) e^{\beta\left(b-x_{1}\right) t} d t=C_{1} \int_{0}^{\infty} \beta t \eta(t) e^{-\beta\left(b-x_{1}\right) t} d t-e^{x_{1}}(1+K) .
\end{array}\right.
$$

Canceling $C_{2}$, we attain

$$
\begin{aligned}
& \left(\int_{0}^{\infty} \eta(t) e^{\beta\left(b-x_{1}\right) t} d t\right)^{-1}\left\{C_{1} \int_{0}^{\infty} \eta(t) e^{-\beta\left(b-x_{1}\right) t} d t-e^{x_{1}}(1+K)\right\} \\
& =\left(\int_{0}^{\infty}-\beta t \eta(t) e^{\beta\left(b-x_{1}\right) t} d t\right)^{-1}\left\{C_{1} \int_{0}^{\infty} \beta t \eta(t) e^{-\beta\left(b-x_{1}\right) t} d t-e^{x_{1}}(1+K)\right\} .
\end{aligned}
$$

where $C_{1}=e^{x_{2}}(1+K)\left(\int_{0}^{\infty} \eta(t) e^{\beta\left(b-x_{2}\right) t} d t\right)^{-1}$. The solution $x_{1}$ of the above equation also gives $C_{2}$ by plugging $x_{1}$ into $(7)$, and we have $v_{1}(x)$.

\section{Verification Theorem}

In this section, we give a verification theorem to show that the solutions $v_{i}(x)$ of (5) are equal to the value functions $V_{i}(x)$ for $i=1,2$ respectively, and the sequences of optimal stopping times are given by $\left(x_{1}, x_{2}\right)$.

Theorem 4.1. Let $\left(x_{1}, x_{2}\right)$ be a solution to (8) and (10) satisfying

$$
x_{1} \leq \frac{1}{a}\left(\frac{\sigma^{2}}{2}+a b-\rho\right) \leq x_{2} \quad \text { and } \quad x_{2}-x_{1}>\log \left(\frac{1+K}{1-K}\right) .
$$

Let

$$
\left\{\begin{array}{c}
v_{1}(x)=\left\{\begin{array}{cl}
C_{2} \int_{0}^{\infty} \eta(t) e^{\beta(b-x) t} d t, & \text { if } x \geq x_{1}, \\
C_{1} \int_{0}^{\infty} \eta(t) e^{-\beta(b-x) t} d t-e^{x}(1+K), & \text { if } x<x_{1},
\end{array}\right. \\
v_{2}(x)=\left\{\begin{array}{cc}
C_{1} \int_{0}^{\infty} \eta(t) e^{-\beta(b-x) t} d t, & \text { if } x<x_{2}, \\
e^{x}(1-K), & \text { if } x \geq x_{2},
\end{array}\right.
\end{array}\right.
$$

with $C_{1}, C_{2}$ which are determined by $\left(x_{1}, x_{2}\right)$ as in (9). If the following inequalities

$$
\left\{\begin{array}{l}
v_{1}(x) \geq v_{2}-e^{x}(1+K), \\
v_{2}(x) \geq e^{x}(1-K)
\end{array}\right.
$$

hold on the interval $\left(x_{1}, x_{2}\right)$, then

$$
v_{i}(x)=V_{i}(x)
$$


for $i=1,2$. Moreover, let

$$
\Lambda_{1}^{*}=\left\{\tau^{*}, \delta^{*}\right\}
$$

where the stopping times $\tau^{*}=\inf \left\{t \geq 0: X(t) \leq x_{1}\right\}, \delta^{*}=\inf \left\{t \geq \tau^{*}: X(t) \leq x_{2}\right\}$, and

$$
\Lambda_{2}^{*}=\left\{\delta^{*}\right\}
$$

where the stopping times $\delta^{*}=\inf \left\{t \geq 0: X(t) \leq x_{2}\right\}$. Then $\Lambda_{1}^{*}$ and $\Lambda_{2}^{*}$ are optimal.

To prove the theorem, we need the following lemma.

Lemma 4.2. Given $z_{1}$ and $z_{2}$, let $\theta_{1}=\inf \left\{t: X(t) \leq z_{1}\right\}$ and $\theta_{2}=\inf \{t: X(t) \geq$ $\left.z_{2}\right\}$. Then

$$
P\left(\theta_{1}<\infty\right)=P\left(\theta_{2}<\infty\right)=1 .
$$

Proof. See the proof of Lemma 6 in [10].

Proof of the Theorem. We need two steps to prove. First, we show that $v_{i}(x) \geq$ $J_{i}\left(x, \Lambda_{i}\right)$ for all $\Lambda_{i}, i=1,2$. As the second, we show that $v_{i}(x)=J_{i}\left(x, \Lambda_{i}^{*}\right)$. Therefore, $v_{i}(x)=V_{i}(x)$ and $\Lambda_{i}^{*}$ is optimal.

It is easy to show that $v_{1}(x) \geq 0$ on $\left(x_{1}, \infty\right)$ since $C_{2}>0$ in (6). Note that $v_{1} \in C^{2}\left(\mathbb{R} \backslash\left\{x_{1}\right\}\right)$ and $v_{2} \in C^{2}\left(\mathbb{R} \backslash\left\{x_{2}\right\}\right)$ satisfying

$$
\left\{\begin{array}{l}
\rho v_{i}(x)-\mathcal{M} v_{i}(x) \geq 0 \\
\left.v_{1}(x) \geq v_{2}(x)-e^{x}(1+K)\right\} \\
v_{2}(x) \geq e^{x}(1-K)
\end{array}\right.
$$

for all $x \in \mathbb{R}$ and,

$$
\begin{cases}\rho v_{1}(x)-\mathcal{M} v_{1}(x) \geq 0, & \text { on }\left(x_{1}, \infty\right), \\ \left.v_{1}(x) \geq v_{2}(x)-e^{x}(1+K)\right\}, & \text { on }\left(-\infty, x_{1}\right), \\ \rho v_{2}(x)-\mathcal{M} v_{2}(x) \geq 0, & \text { on }\left(-\infty, x_{2}\right), \\ v_{2}(x) \geq e^{x}(1-K), & \text { on }\left(x_{2}, \infty\right) .\end{cases}
$$

Using $\rho v_{i}(x)-\mathcal{M} v_{i}(x) \geq 0$, Dynkin's formula and Fatou's lemma as in Øksendal (2003, p. 226), for any stopping times $0 \leq \theta_{1} \leq \theta_{2}$, we have

$$
E e^{-\rho \theta_{1}} v_{i}\left(X_{\theta_{1}}\right) \geq E e^{-\rho \theta_{2}} v_{i}\left(X_{\theta_{2}}\right)
$$

a.s., for $i=1$ or 2 . Given $\Lambda_{1}=(\tau, \delta)$, we have

$$
\begin{aligned}
v_{1}(x) & \geq E e^{-\rho \tau} v_{1}(X(\tau)) \\
& \geq E e^{-\rho \tau}\left(v_{2}(X(\tau))-S(\tau)(1+K)\right) \\
& =E e^{-\rho \tau}\left(v_{2}(X(\tau))-E e^{-\rho \tau} S(\tau)(1+K)\right. \\
& \geq E e^{-\rho \delta}[S(\delta)(1-K)]-E e^{-\rho \tau}[S(\tau)(1+K)] \\
& =E\left[e^{-\rho \delta} S(\delta)(1-K)-e^{-\rho \tau} S(\tau)(1+K)\right]
\end{aligned}
$$

from (2) and (11). We have $v_{1}(x) \geq J_{1}\left(x, \Lambda_{1}\right)$ which implies that $v_{1}(x) \geq V_{1}(x)$. 
Similarly, we can show that $v_{2}(x) \geq V_{2}(x)$.

Next, we construct the equalities. Define a stopping time

$$
\tau^{*}=\left\{\begin{array}{cc}
0 & \text { if } x \leq x_{1} \\
\inf \left\{t \geq 0: X(t)=x_{1}\right\}, & \text { if } x>x_{1}
\end{array}\right.
$$

Then, $\tau^{*}<\infty$, a.s. by Lemma 4.2. Using Dynkin's formula, we have

$$
\begin{aligned}
v_{1}(x) & =E e^{-\rho \tau^{*}} v_{1}\left(X\left(\tau^{*}\right)\right) \\
& =E e^{-\rho \tau^{*}}\left(v_{2}\left(X\left(\tau^{*}\right)\right)-S\left(\tau^{*}\right)(1+K)\right) \\
& =E e^{-\rho \tau^{*}}\left(v_{2}\left(X\left(\tau^{*}\right)\right)-E e^{-\rho \tau^{*}} S\left(\tau^{*}\right)(1+K) .\right.
\end{aligned}
$$

Let $\delta^{*}=\inf \left\{t \geq \tau^{*}: X(t)=x_{2}\right\}$. Then, $\delta^{*}<\infty$ a.s. by Lemma 4.2, and

$$
\begin{aligned}
E e^{-\rho \tau^{*}} v_{2}\left(X\left(\tau^{*}\right)\right) & =E e^{-\rho \delta^{*}} v_{2}\left(X\left(\delta^{*}\right)\right) \\
& =E e^{-\rho \delta^{*}}\left(S\left(\delta^{*}\right)(1-K)\right)
\end{aligned}
$$

Therefore, it follows that

$$
v_{1}(x)=E\left[e^{-\rho \delta^{*}} S\left(\delta^{*}\right)(1-K)-e^{-\rho \tau^{*}} S\left(\tau^{*}\right)(1+K)\right] .
$$

and it is positive by the second hypothesis of this theorem.

Similarly, we have

$$
v_{2}(x)=E\left[e^{-\rho \delta^{*}} S\left(\delta^{*}\right)(1-K)\right] .
$$

This completes the proof.

\section{A Numerical Example}

In this section, we demonstrate a numerical example with the following:

$$
a=0.8, \quad b=2, \quad \sigma=0.5, \quad \rho=0.01, \quad K=0.01
$$

As the result of solving $(7)$, we have $\left(x_{1}, x_{2}\right)=(1.5058,2.2434)$. It implies that the low stock price $S_{1}=\exp x_{1}=4.5078$ and the high price $S_{2}=\exp x_{2}=9.4253$.

The probability or the frequency for the price to increase from $S_{1}$ to $S_{2}$ are closely connected to the price levels $S_{1}$ and $S_{2}$.

The corresponding value functions $V_{1}(x)$ and $V_{2}(x)$ are plotted in Figure 2. As we see in the picture, $V_{1}(x)$ is uniformly bounded and $V_{2}(x)$ is increasing with an exponential growth rate.

Now we vary each parameters at a time and observe the dependence of $\left(x_{1}, x_{2}\right)$. First we vary the equilibrium parameter $b$ to get the associated $\left(x_{1}, x_{2}\right)$. Intuitively, larger $b$ would result larger rewards and larger threshold pairs $\left(x_{1}, x_{2}\right)$. Table 1 confirms this observation. For the monotonely increased $b$, the result gives the 

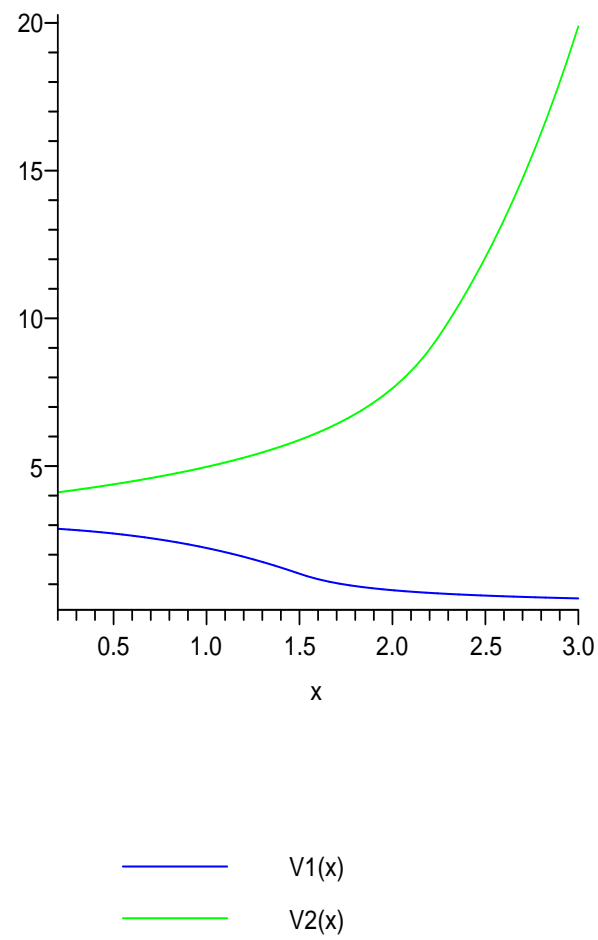

Figure 2. The value functions $V_{1}(x)$ and $V_{2}(x)$

\begin{tabular}{|c|c|c|c|c|c|}
\hline$b$ & 1 & 1.5 & 2 & 2.5 & 3 \\
\hline$x_{1}$ & 0.5058 & 1.0058 & 1.5058 & 2.0058 & 2.5058 \\
\hline$x_{2}$ & 1.2434 & 1.7434 & 2.2434 & 2.7434 & 3.2434 \\
\hline$V_{1}(1)$ & 0.2935 & 0.8226 & 2.2266 & 4.4785 & 7.9976 \\
\hline$V_{2}(1)$ & 2.8066 & 3.5680 & 4.9721 & 7.2239 & 10.7431 \\
\hline
\end{tabular}

Table 1. The thresholds $\left(x_{1}, x_{2}\right)$ for varying $b$

monotonely increased $\left(x_{1}, x_{2}\right)$. We added the values $\left(V_{1}(1), V_{2}(1)\right)$ to observe the values of the associated value functions.

Next, we vary the rate of reversion $a$ in Table 2. A larger a implies smaller difference between the threshold levels $\left(x_{1}, x_{2}\right)$. Because the oscillating of the stock price tends to bounce more narrowly on a range for a larger $a$, it is natural that the gap between buying price and selling price is decreased.

In Table 3, we vary the volatility $\sigma$. Larger $\sigma$ implies greater range for the stock price $S(t)=\exp (x(t))$. Intuitively larger volatility makes further gap between a high price of stock and a low price of stock, and it is showed this as well. 


\begin{tabular}{|c|c|c|c|c|c|}
\hline$a$ & 0.6 & 0.7 & 0.8 & 0.9 & 1 \\
\hline$x_{1}$ & 1.5015 & 1.5026 & 1.5058 & 1.5099 & 1.5143 \\
\hline$x_{2}$ & 2.3695 & 2.2991 & 2.2434 & 2.1982 & 2.1606 \\
\hline$V_{1}(1)$ & 2.6378 & 2.4044 & 2.2266 & 2.0860 & 1.9714 \\
\hline$V_{2}(1)$ & 5.3827 & 5.1498 & 4.9721 & 4.8314 & 4.7169 \\
\hline
\end{tabular}

Table 2. $\left(x_{1}, x_{2}\right)$ with varying $a$

\begin{tabular}{|c|c|c|c|c|c|}
\hline$\sigma$ & 0.3 & 0.4 & 0.5 & 0.6 & 0.7 \\
\hline$x_{1}$ & 1.5531 & 1.5242 & 1.5058 & 1.5031 & 1.5177 \\
\hline$x_{2}$ & 1.9374 & 2.0848 & 2.2434 & 2.4134 & 2.5928 \\
\hline$V_{1}(1)$ & 1.3231 & 1.7414 & 2.2266 & 2.8003 & 3.4828 \\
\hline$V_{2}(1)$ & 4.0686 & 4.4869 & 4.9721 & 5.5475 & 6.2282 \\
\hline
\end{tabular}

Table 3. $\left(x_{1}, x_{2}\right)$ with varying $\sigma$

\begin{tabular}{|c|c|c|c|c|c|}
\hline$\rho$ & 0.001 & 0.005 & 0.01 & 0.015 & 0.02 \\
\hline$x_{1}$ & 1.5080 & 1.5071 & 1.5058 & 1.5045 & 1.5032 \\
\hline$x_{2}$ & 2.2541 & 2.2494 & 2.2434 & 2.2375 & 2.2316 \\
\hline$V_{1}(1)$ & 2.2813 & 2.2569 & 2.2266 & 2.1966 & 2.1669 \\
\hline$V_{2}(1)$ & 5.0268 & 5.0024 & 4.9721 & 4.9421 & 4.9123 \\
\hline
\end{tabular}

Table 4. $\left(x_{1}, x_{2}\right)$ with varying $\rho$

\begin{tabular}{|c|c|c|c|c|c|}
\hline$K$ & 0.001 & 0.005 & 0.01 & 0.015 & 0.02 \\
\hline$x_{1}$ & 1.5284 & 1.5183 & 1.5058 & 1.4936 & 1.4815 \\
\hline$x_{2}$ & 2.2434 & 2.2434 & 2.2434 & 2.2434 & 2.2434 \\
\hline$V_{1}(1)$ & 2.2963 & 2.2653 & 2.2266 & 2.1879 & 2.1492 \\
\hline$V_{2}(1)$ & 5.0173 & 4.9972 & 4.9721 & 4.9470 & 4.9218 \\
\hline
\end{tabular}

Table 5. $\left(x_{1}, x_{2}\right)$ with varying $K$

In Table 4, we vary the discount rate $\rho$. Large $\rho$ implies smaller reward functions, and smaller $\left(x_{1}, x_{2}\right)$ and Table 4 shows that.

As the last, we vary the slippage rate $K$ in Table 5 . The result shows that $x_{1}$ is monotonely decreasing in $K$ and $x_{2}$ stays constantly. This is because lager $K$ discourages stock translations and has to be returned by smaller $x_{1}$. Comparing with the result of the paper [10] under the same condition, we got the result $\left(x_{1}, x_{2}\right)=$ $(1.1998,1.6385)$ whose $x_{1}$ level is slightly less than the result in [10]. It's because our $V_{1}(x)$ doesn't associate with $V_{1}(x)$, and this makes $V_{2}(x)$ smaller, so even $V_{1}(x)$. Indeed, our $\left(V_{1}(1), V_{2}(1)\right)=(0.3846,3.1303)$ which are both less than the values of $\left(V_{1}(1), V_{2}(1)\right)$ in [10] whose value of $\left(V_{1}(1), V_{2}(1)\right)$ was $(0.607,3.364)$ under the 
five variables condition $a=0.8, b=2, \sigma=0.5, \rho=0.5, K=0.01$. In practice, the profit function having one trade is obviously smaller than the profit function having infinitive trades. In advance, studying about a problem having finite multiple trades, or applying this result to real data should be interesting subjects.

\section{Conclusion}

Through this paper, we solved quasi-algebraic equations for the two threshold levels. We showed that the two thresholds lead to the optimal stopping times to buy and sell a share.

\section{REFERENCES}

1. Eloe, P., Liu R.H., Yatsuki M., Yin, G. \& Zhang, Q.: Optimal selling rules in a regimeswitching exponential Gaussian diffusion model. SIAM J. Appl. Math. 69 (2008), no. 3, 810-829.

2. Guo, X. \& Zhang, Q.: Closed-form solutions for perpetual american put options with regime switching. SIAM J. Appl. Math. 64 (2004), 2034-2049.

3. Fama, E. \& French, K.: Forecasting Profitability and Earnings. J. of Business 73 (2000), no $2,161-175$.

4. Frankel, J. \& Rose, A.: A panel project on purchasing power parity: Mean reversion within and between countries. J. of International Economics 40 (1996), 209-224.

5. McKean, H.P.: A free boundary problem for the heat equation arising from a problem of mathematical economics. Inderstrial Managem. Review 61 (Spring, 1965), 32-39.

6. Shin, D.: American perpetual put option valuation with one time regime switching. preprint, 2009.

7. Song, Q.S., Yin, G. \& Zhang, Q.: Stochastic Optimization Methods for Buying-Lowand-selling-High Strategies. Stochastic Analysis and Applications 27 (2009), no. 3, 523542.

8. Uhlenbeck, G.E. \& Ornstein, L.S.: On the theory of Brownian Motion. Physical Review 36 (1930), 823-841.

9. Poterba, J. \& Summers, L.: Mean Reversion in Stock Prices: Evidence and Implication. NBER Working paper 2343 (August, 1987).

10. Zhang, H. \& Zhang, Q.: Trading a mean-reverting asset: Buy law and sell high. Automatica 44 (June, 2008), 1511-1518.

Institute for Quantitative Finance and Technology, Korea University, Seoul, 136701, Korea

Email address: theater7@korea.ac.kr 